\title{
Blood pressure prior to percutaneous coronary intervention is associated with the risk of end-stage renal disease: a nationwide population based-cohort study
}

\author{
Eun Hui Bae' ${ }^{1}$, Sang Yup Lim², Bongseong Kim³ ${ }^{3}$ Kyung-Do Han ${ }^{3}$, Tae Ryom Oh${ }^{1}$, Hong Sang Choi ${ }^{1}$, \\ Chang Seong Kim ${ }^{1}$, Seong Kwon $\mathrm{Ma}^{1}$, Soo Wan Kim ${ }^{1}$ \\ ${ }^{1}$ Department of Internal Medicine, Chonnam National University Medical School, Gwangju, Republic of Korea \\ ${ }^{2}$ Department of Internal Medicine, Korea University Ansan Hospital, Ansan, Republic of Korea \\ ${ }^{3}$ Department of Statistics and Actuarial Science, Soongsil University, Seoul, Republic of Korea
}

Background: Hypertension is the most important modifiable risk factor for mortality and morbidity in chronic kidney disease and coronary artery syndrome. The effect of hypertension prior to percutaneous coronary intervention ( $\mathrm{PCl}$ ) on the development of end-stage renal disease (ESRD) is unknown.

Methods: We used nationally representative data from the Korean National Health Insurance System-140,164 subjects were enrolled during 2010-2015; they were free of ESRD at enrolment, underwent PCI, and were followed up until 2017. Blood pressure (BP) was measured within at least 2 years prior to PCl. The primary outcome was the development of ESRD.

Results: During a median follow-up of 5.4 years, 2,082 participants (1.5\%) developed ESRD. The highest systolic BP group (>160 $\mathrm{mmHg}$ ) showed a higher hazard ratio (3.69; 95\% confidence interval, 2.61-5.23) than the reference group (110-119 $\mathrm{mmHg}$ ). Similar results were observed in the highest diastolic BP group $(>120 \mathrm{mmHg})$, which showed a higher hazard ratio than the reference group (70-79 mmHg). However, ESRD risk showed a J-shaped relationship with baseline systolic and diastolic BP at 113 and 74 $\mathrm{mmHg}$ in diabetes mellitus subgroup, respectively, after adjustment for potential confounders.

Conclusion: Our study showed that a high systolic or diastolic BP prior to $\mathrm{PCl}$ was independently associated with an increased incidence of ESRD.

Keywords: End-stage renal disease, Hypertension, Korean, Percutaneous coronary intervention, Risk

Received: December 2, 2020; Revised: April 6, 2021; Accepted: April 21, 2021

Editor: Sungjin Chung, The Catholic University of Korea, Seoul, Republic of Korea

Correspondence: Soo Wan Kim

Department of Internal Medicine, Chonnam National University Medical School, 42 Jebongro, Gwangju 61469, Republic of Korea.

E-mail: skimw@chonnam.ac.kr

ORCID: https://orcid.org/0000-0002-3540-9004

Eun Hui Bae and Sang Yup Lim contributed equally to this study as co-first authors.

Copyright (C) 2021 by The Korean Society of Nephrology

(a) This is an Open Access article distributed under the terms of the Creative Commons Attribution Non-Commercial and No Derivatives License (http:// creativecommons.org/licenses/by-nc-nd/4.0/) which permits unrestricted non-commercial use, distribution of the material without any modifications, and reproduction in any medium, provided the original works properly cited. 


\section{Introduction}

Hypertension is the most important modifiable risk factor globally for overall mortality and morbidity [1]. Hypertension also plays a crucial role in the development and progression to end-stage renal disease (ESRD) [2,3]. Observational studies have shown that death due to cardiovascular (CV) disease increases progressively and linearly with blood pressure (BP) $[4,5]$. However, this linear theory has been challenged for nearly three decades, especially for diastolic BP (DBP) [6-9]. Physiologically, a J-shaped or U-shaped curve phenomenon would be expected to exist in vital signs such as BP and other biological signs, with increased mortality exhibited at both ends of the spectrum. The linear relationship might be true for the general population but not for patients with chronic illness. In particular, after an acute coronary syndrome, a J- or U-shaped association has been shown between $\mathrm{BP}$ and the risk of $\mathrm{CV}$ events $[10,11]$.

Percutaneous coronary intervention (PCI) is an essential treatment modality for coronary artery disease, and following advancements in PCI techniques, its indications and applications have been widening. Although PCI is mainly performed in patients with underlying conditions such as diabetes mellitus (DM), chronic kidney disease (CKD), and hypertension, resulting in ESRD, data regarding the association between BP prior to PCI and ESRD remain insufficient. In addition, the association between $\mathrm{BP}$ values prior to PCI and ESRD risk has not been evaluated.

To evaluate the role of BP as a predictor of incident ESRD in patients undergoing PCI, we analyzed nationally representative data from the Korean National Health Insurance System.

\section{Methods}

Because of the confidentiality of the data used for this study and strict privacy policy from the data holder restricting data access and use to the designated research personnel only, the data cannot be provided to other people, whether or not the data are made anonymous.

\section{Study design and database}

The Korean National Health Insurance Service (KNHIS) comprises a complete set of health information of 50 mil- lion Koreans, including an eligibility database, a medical treatment database, a health examination database, and a medical care institution database [12-14]. The National Health Insurance Corporation (NHIC) is the single insurer, managed by the Korean government, to which approximately $97 \%$ of the Korean population subscribes. Enrollees in the NHIC are recommended to undergo a standardized medical examination at least every 2 years. Among 270,237 subjects who underwent PCI from 2010 to 2015 (index year), 143,981 subjects were followed up to 31 December 2017. We excluded 2,440 subjects with missing data for at least one variable. To avoid confounding effects of preexisting diseases and minimize the possible effects of reverse causality, those with a history of ESRD before the index year were also excluded ( $\mathrm{n}=1,123)$. Ultimately, the study population comprised 140,164 subjects (Fig. 1). We registered only de novo PCI and excluded patients with a history of PCI to avoid the effects of past coronary intervention due to coronary artery disease, including angina pectoris or myocardial infarction.

This study was approved by the Institutional Review Board

270,237 people underwent $\mathrm{PCl}$ in the Korean $\mathrm{NHI}$ database in 2010-2015

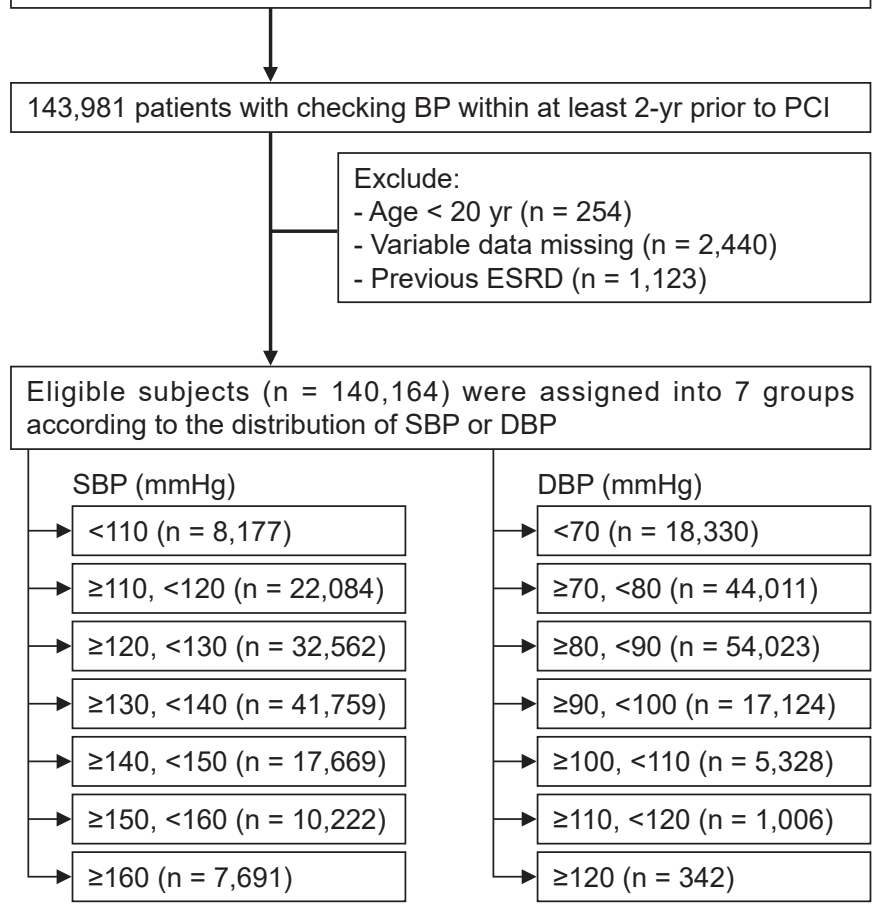

Figure 1. Flow diagram of the study.

$\mathrm{BP}$, blood pressure; DBP, diastolic BP; ESRD, end-stage renal disease; NHI, National Health Insurance; $\mathrm{PCl}$, percutaneous coronary intervention; SBP, systolic BP. 
(IRB) of Chonnam National University Hospital (No. CNUHEXP-2019-035) and National Health Insurance Service (NHIS-2019-1-379), and it was conducted according to the principles of the Declaration of Helsinki. The need for written informed consent was waived by the IRB.

\section{Measurements and definitions}

In the KNHIS, the equipment used to measure BP varies between sites. However, most people received their medical examinations in the same hospital near their residence, and most BP measurements were performed using the same equipment in each individual. BP was measured by trained clinicians. Systolic BP (SBP) and DBP were measured, and the sitting brachial $\mathrm{BP}$ was the average of the two measurements taken after the subject had been seated for 5 minutes with an arm in the appropriate position. Body mass index (BMI) was calculated as the subject's weight in kilograms divided by the square of the subject's height in meters. Information on current smoking and alcohol consumption was obtained by a questionnaire. Based on alcohol consumption status, participants were categorized as non-drinker, mild drinker ( $<30 \mathrm{~g} /$ day), or heavy drinker ( $\geq 30 \mathrm{~g} /$ day). Regular exercise was defined as physical activity that was performed at least five times per week. Income level was dichotomized at the lower $25 \%$. Blood samples for the measurement of serum glucose and total cholesterol levels were drawn after an overnight fast. Proteinuria was tested by the dipstick method and defined as negative, trace, and 1+ to 4+. Comorbidities were identified using information gathered in the 1 year before the index date. Hypertension was defined as a previous hypertension diagnosis International Classification of Diseases (ICD)-10 codes (I10-13, I15) and a history of taking at least one antihypertensive drug, a recorded SBP of $\geq 140$ $\mathrm{mmHg}$, or DBP of $\geq 90 \mathrm{mmHg}$ in the health examination database. DM was identified using the appropriate diagnostic codes (E11-14) and medical history of DM or a recorded fasting serum glucose concentration of $\geq 126 \mathrm{mg} / \mathrm{dL}$ in the health examination database. Dyslipidemia was identified using the appropriate diagnostic code (E78) and history of lipid-lowering drug use or a total serum cholesterol concentration of $\geq 240 \mathrm{mg} / \mathrm{dL}$ in the health examination database. CKD was defined as an estimated glomerular filtration rate (eGFR) of $<60 \mathrm{~mL} / \mathrm{min} / 1.73 \mathrm{~m}^{2}$ calculated using the CKD Epidemiology Collaboration (CKD-EPI) equation. The par- ticipants' fasting blood glucose $(\mathrm{mg} / \mathrm{dL})$, total cholesterol $(\mathrm{mg} / \mathrm{dL})$, triglyceride $(\mathrm{mg} / \mathrm{dL})$, high-density lipoprotein cholesterol (mg/dL), and low-density lipoprotein cholesterol $(\mathrm{mg} / \mathrm{dL})$ concentrations were measured in a fasting state. The quality of the laboratory tests has been warranted by the Korean Association for Laboratory Medicine, and the hospitals participating in the National Health Insurance health checkup programs are certified by the NHIS.

\section{Study outcomes and follow-up}

The study population was followed from baseline to the date of ESRD diagnosis or until December 31, 2017, whichever came first. The primary endpoint was incident ESRD, which was defined using a combination of ICD-10 codes (N18-19, Z49, Z94.0, and Z99.2) and a unique code (V code) that was assigned in the initiation of renal replacement therapy (hemodialysis [HD], V001; peritoneal dialysis [PD], V003) and/ or kidney transplantation (KT, V005) during hospitalization. All medical expenses for dialysis are reimbursed using the Korean Health Insurance Review and Assessment Service database. These patients are also registered as special medical aid beneficiaries. Therefore, we identified every patient with ESRD in the entire South Korean population and analyzed the data for all patients with ESRD who started dialysis. Codes for treatment or medical expense claims included V005 for KT, V001 for HD, and V003 for PD. We excluded individuals without previous CKD who had a transplant or dialysis code on the same date as an acute renal failure code. Subjects on continuous renal replacement therapy or acute PD were also excluded.

\section{Statistical analysis}

We report the mean \pm standard deviation with intervals for continuous variables and the numbers (with percentages) for categorical variables. Participants were classified into seven groups according to the SBP and DBP levels. To identify the risk of ESRD by SBP and DBP level, we calculated the hazard ratios (HRs) with $95 \%$ confidence intervals (CIs) and analyzed these data using the Cox proportional hazard regression model. We analyzed associations between BP level and ESRD development using three models: model 1 , crude model; model 2, adjusted for model 1 plus age, sex, income, DM, dyslipidemia, and hypertension; model 3, adjusted for 
model 2 plus smoking, alcohol drinking, physical activity, and eGFR. We also performed subgroup analysis for DM and CKD. A p-value of $<0.05$ was considered to reflect statistical significance. SAS version 9.3 software and SAS survey procedures (SAS Institute, Inc., Cary, NC, USA) were used for all statistical analyses.

\section{Results}

\section{Baseline characteristics}

Table 1 shows the baseline characteristics of the participants regarding the development of ESRD. Among all the participants, 2,082 (1.5\%) developed ESRD during a median follow-up duration of 5.4 years. The mean age was higher among individuals who developed ESRD than among those who did not. The proportion of low income was higher in the incident ESRD group than in the non-ESRD group. Comorbidities such as DM, hypertension, dyslipidemia, CKD, and proteinuria were more prevalent in the ESRD group than in the non-ESRD group. GFR and BMI were lower, while BP and glucose levels were higher in the ESRD group than in the non-ESRD group (Table 1).

The characteristics of participants classified by levels of SBP and DBP are presented in Tables 2 and 3, respectively. Subjects in the high SBP group were older, were more likely to be women, had a lower income, exercised less, and had a higher prevalence of DM and CKD (Table 2). BMI, waist circumference, and fasting glucose were also higher in the high SBP group. Lipid profile was higher in the high SBP group (Table 2). Subjects in the higher DBP group were younger, were more likely to be men, had a low income, exercised

Table 1. Baseline characteristics of subjects according to the incident ESRD

\begin{tabular}{|c|c|c|c|}
\hline Characteristic & Non-ESRD & ESRD & p-value \\
\hline No. of patients & 138,082 & 2,082 & \\
\hline Age (yr) & $63.4 \pm 10.6$ & $65.4 \pm 9.9$ & $<0.001$ \\
\hline Male sex & 97,897 (70.9) & $1,451(69.7)$ & 0.23 \\
\hline Current smoker & $41,174(29.8)$ & $539(25.9)$ & $<0.001$ \\
\hline Heavy drinker & $8,958(6.5)$ & $76(3.7)$ & $<0.001$ \\
\hline Regular exercise & $28,386(20.6)$ & 355 (17.1) & $<0.001$ \\
\hline Income-low & $29,846(21.6)$ & $552(26.5)$ & $<0.001$ \\
\hline Diabetes mellitus & $44,078(31.9)$ & $1,562(75.0)$ & $<0.001$ \\
\hline Hypertension & 95,743 (69.3) & $1,945(93.4)$ & $<0.001$ \\
\hline Dyslipidemia & $70,411(51.0)$ & 1,373 (65.9) & $<0.001$ \\
\hline $\mathrm{CKD}^{\mathrm{a}}$ & $19,562(14.2)$ & $1,659(79.7)$ & $<0.001$ \\
\hline Proteinuria & & & $<0.001$ \\
\hline Negative & $125,014(91.2)$ & $728(35.3)$ & \\
\hline Trace & $4,326(3.2)$ & $88(4.3)$ & \\
\hline $1+$ & $4,361(3.2)$ & $296(14.4)$ & \\
\hline $2+$ & 2,407 (1.8) & $482(23.4)$ & \\
\hline $3+$ & $805(0.6)$ & 369 (17.9) & \\
\hline $4+$ & $152(0.1)$ & $97(4.7)$ & \\
\hline GFR $\left(\mathrm{mL} / \mathrm{min} / 1.73 \mathrm{~m}^{2}\right)$ & $81.8 \pm 40.8$ & $41.3 \pm 27.7$ & $<0.001$ \\
\hline Body mass index $\left(\mathrm{kg} / \mathrm{m}^{2}\right)$ & $24.7 \pm 3.0$ & $24.5 \pm 3.2$ & 0.03 \\
\hline Glucose (mg/dL) & $112.2 \pm 38.1$ & $136.0 \pm 67.9$ & $<0.001$ \\
\hline $\mathrm{TC}(\mathrm{mg} / \mathrm{dL})$ & $205.2 \pm 46.2$ & $203.6 \pm 57.2$ & 0.11 \\
\hline $\mathrm{SBP}(\mathrm{mmHg})$ & $130.1 \pm 15.9$ & $137.5 \pm 19.6$ & $<0.001$ \\
\hline $\mathrm{DBP}(\mathrm{mmHg})$ & $79.0 \pm 10.3$ & $80.1 \pm 12.1$ & $<0.001$ \\
\hline Follow-up (yr) & $5.5 \pm 1.9$ & $2.5 \pm 2.0$ & $<0.001$ \\
\hline
\end{tabular}

Data are expressed as number only, mean \pm standard deviation, or number (\%).

CKD, chronic kidney disease; DBP, diastolic blood pressure; ESRD, end-stage renal disease; GFR, glomerular filtration rate; SBP, systolic blood pressure; TC, total cholesterol

aEstimated GFR $<60 \mathrm{~mL} / \mathrm{min} / 1.73 \mathrm{~m}^{2}$. 


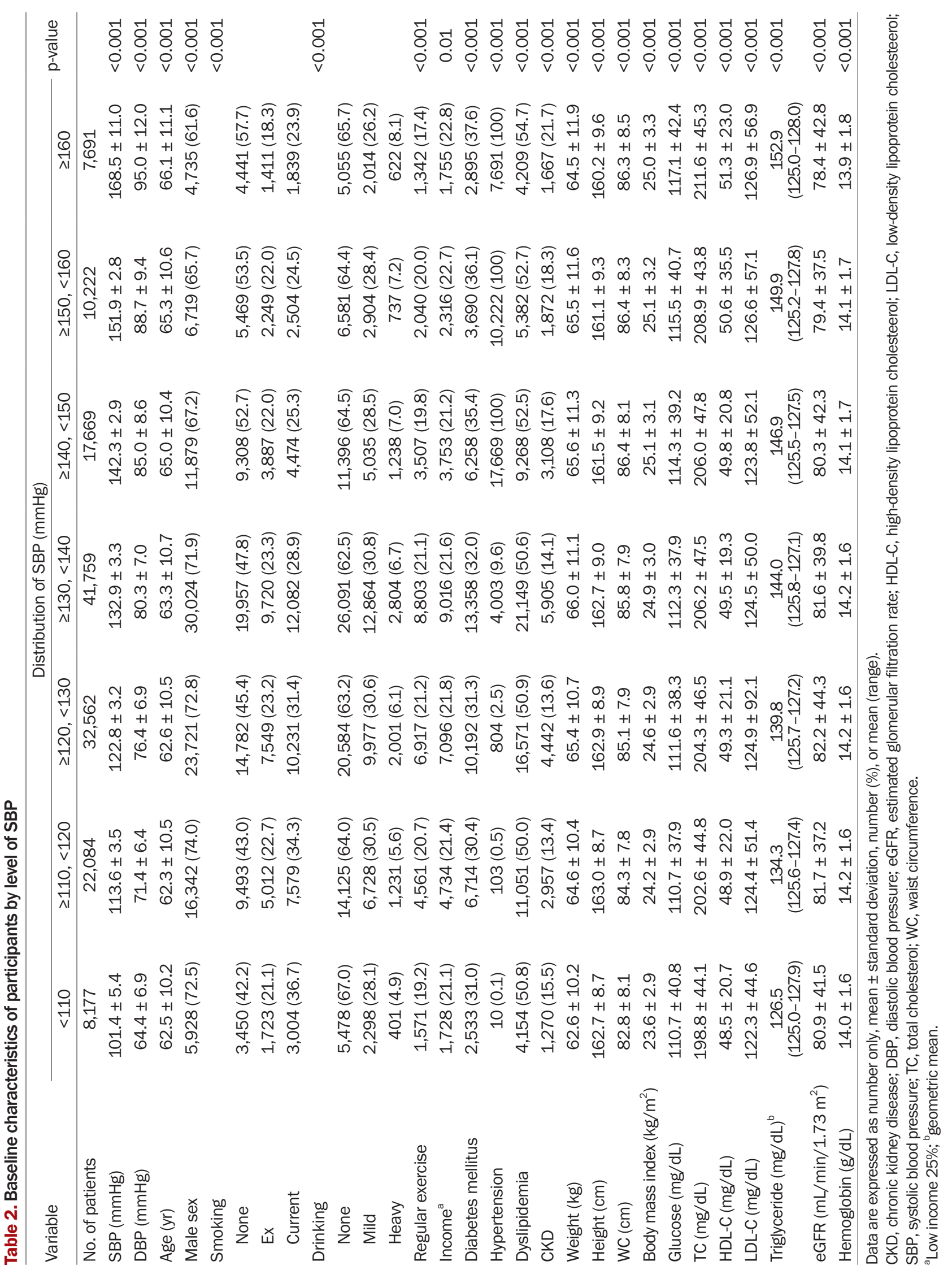




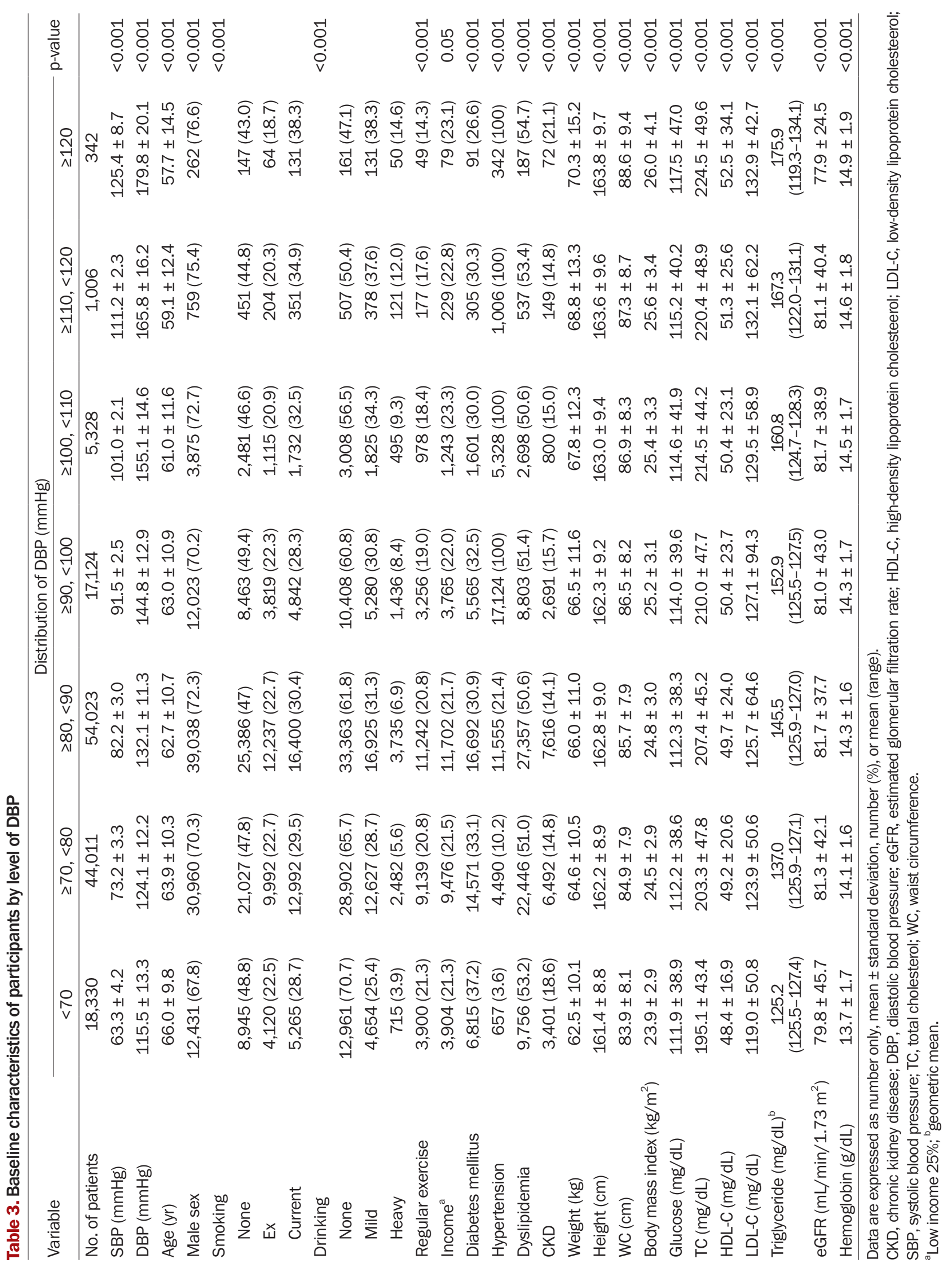


less, and had a higher prevalence of CKD but a lower prevalence of DM (Table 3). BMI, waist circumference, and fasting glucose were also higher in the high SBP group. Lipid profile was higher in the high SBP group (Table 2).

\section{$\mathrm{BP}$ and the risk of ESRD}

During a median follow-up period of 5.4 years after the PCI, 2,082 participants (1.5\%) developed ESRD. The highest SBP group (>160 mmHg) showed the highest HR of 3.69 (95\% CI, 2.61-5.23) compared to the reference group (110-119 $\mathrm{mmHg}$ ). Similar results were observed for the highest DBP group (>120 $\mathrm{mmHg}$ ) as it showed the highest HR compared to the reference group (70-79 mmHg) (Table 4, Fig. 2A, B). The risk of ESRD showed a J-shaped relationship with baseline SBP at $110 \mathrm{mmHg}$ and baseline DBP at $75 \mathrm{mmHg}$ (Table 4; Fig. 3A, B) after adjustment for age, sex, income, presence of DM, dyslipidemia, hypertension, smoking, alcohol drinking, physical activity, and GFR.

\section{Subgroup analyses}

Subgroup analyses for DM and CKD were performed. The DM cases showed a J-shaped curve with a baseline of 113 mmHg for SBP and $74 \mathrm{mmHg}$ for DBP (Fig. 3C, D), while HR for incident ESRD showed a linear relationship with SBP and DBP among the non-DM cases (Fig. 3E, F). In addition, HR for incident ESRD increased only in the DBP > $120 \mathrm{mmHg}$ group (HR, 2.24; 95\% CI, 1.35-3.72) among the non-DM cases. However, the cases with DM showed increased $\mathrm{HR}$ in the DBP $<70$ mmHg group (HR, 1.43; 95\% CI, 1.22-1.68) and the DBP > 120 mmHg group (HR, 2.65; 95\% CI, 1.44-4.88) (Table 5).

Regarding the CKD subgroup analysis, increased HR for incident ESRD was observed in the SBP > $150 \mathrm{mmHg}$ group among the non-CKD cases, while increased HR for incident ESRD was observed in the SBP > $130 \mathrm{mmHg}$ group among the CKD cases (Table 6; Supplementary Fig. 1A, C, available online). For DBP, HR for incident ESRD was increased in the DBP > $120 \mathrm{mmHg}$ group among the non-CKD cases (HR, 5.02; 95\% CI, 1.79-14.09), while the cases with CKD showed a J-shaped curve and increased $\mathrm{HR}$ in the $\mathrm{DBP}<70 \mathrm{mmHg}$ (HR, 1.25; 95\% CI, 1.07-1.46) and DBP 110-119 mmHg groups (HR, 1.69; 95\% CI, 1.13-2.54) (Table 6; Supplementary Fig. 1B, D).

\section{Discussion}

The present study demonstrated that increased levels of both SBP and DBP prior to PCI were associated with a higher risk of ESRD during a 5.4-year follow-up period after undergoing PCI. Both the SBP and DBP levels were associated with the ESRD risk. Moreover, patients with DM undergoing PCI showed a J-shaped relationship with SBP and DBP for ESRD risk. However, patients without DM undergoing PCI showed a linear relationship with SBP and DBP levels for ESRD risk. This association persisted after multivariable adjustment for important potential confounders.

In the last few decades, researchers have mainly focused on finding a target $\mathrm{BP}$ to reduce $\mathrm{CV}$ mortality and improve outcomes. The 2017 American Heart Association/American College of Cardiology hypertension guidelines recommended a target of $<130 / 80 \mathrm{mmHg}$ for patients with ischemic heart disease and DM [15]. The background of this guideline was that the Systolic Blood Pressure Intervention Trial (SPRINT) was prematurely ceased due to the benefits of controlling hypertensive patients' SBP levels to $<120 \mathrm{mmHg}$ rather than $<140 \mathrm{mmHg}$, thus supporting the aim for a low SBP. The composite endpoint of acute coronary syndrome, stroke, acute decompensated heart failure, or death from CV causes was $25 \%$ less likely in those with a target BP of $<120$ mmHg [16]. However, only a few studies have investigated the association of BP and ESRD risk in patients undergoing PCI. Our data showed that the risk of ESRD is associated with increased SBP and DBP. Especially, DBP of PCI with DM group showed a J-shape at a baseline DBP of $74 \mathrm{mmHg}$. Böhm et al. [17] reported a significant increase in adverse events for SBP below $120 \mathrm{mmHg}$, potentially due to poor perfusion leading to an increased risk of ischemic events. White et al. [11] also reported that in patients with type 2 $\mathrm{DM}$ and recent acute coronary syndrome, an average BP of $<130 / 80 \mathrm{mmHg}$ was associated with worsened CV outcomes. Similarly, despite the lack of studies on the adverse effects of low BP on ESRD, our results suggest that low BP is a poor prognostic factor for ESRD in DM patients who underwent PCI. Analyses from other preventive cardiology clinical trials support our findings. The Avoiding Cardiovascular Events Through Combination Therapy in Patients Living with Systolic Hypertension trial also showed similar results. This trial included patients with hypertension and increased CV risk and found that, compared with an SBP of 

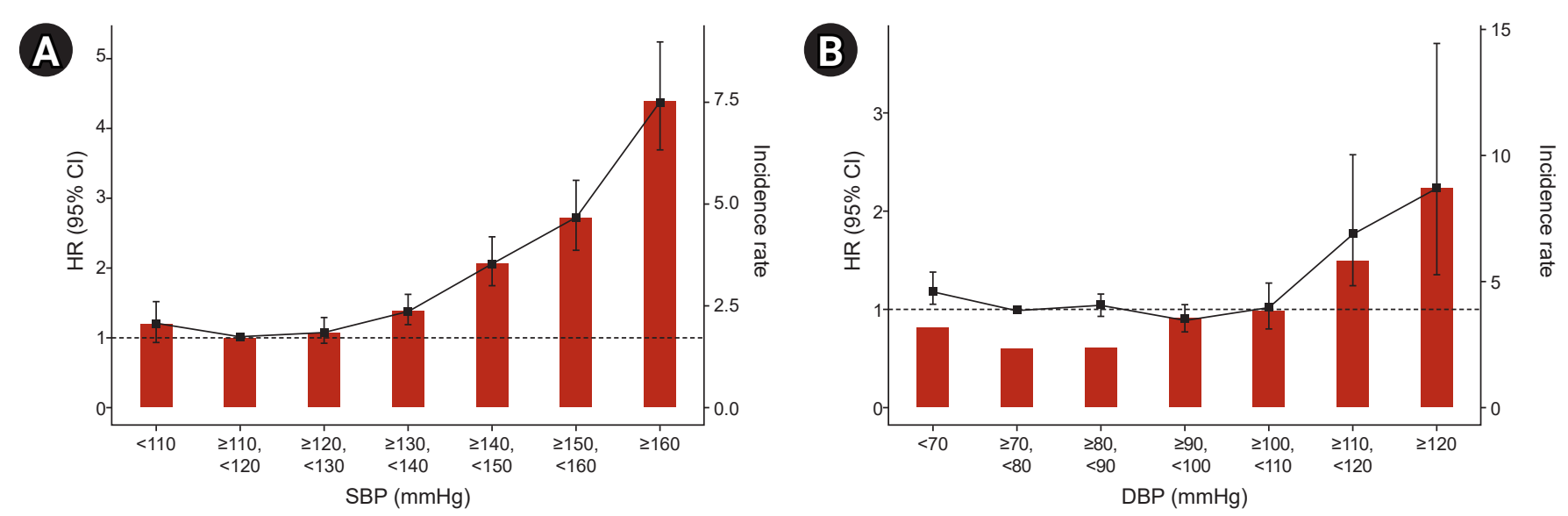

Figure 2. Incidence rates, HRs, and $95 \%$ Cls of ESRD by deciles of SBP (A) and DBP (B). Adjusted for age, sex, income-low $25 \%$, diabetes mellitus, hypertension, dyslipidemia, current smoker, alcohol consumption, regular exercise, and estimated glomerular filtration rate.

$\mathrm{Cl}$, confidence interval; DBP, diastolic blood pressure; ESRD, end-stage renal disease; HR, hazard ratio; SBP, systolic blood pressure.
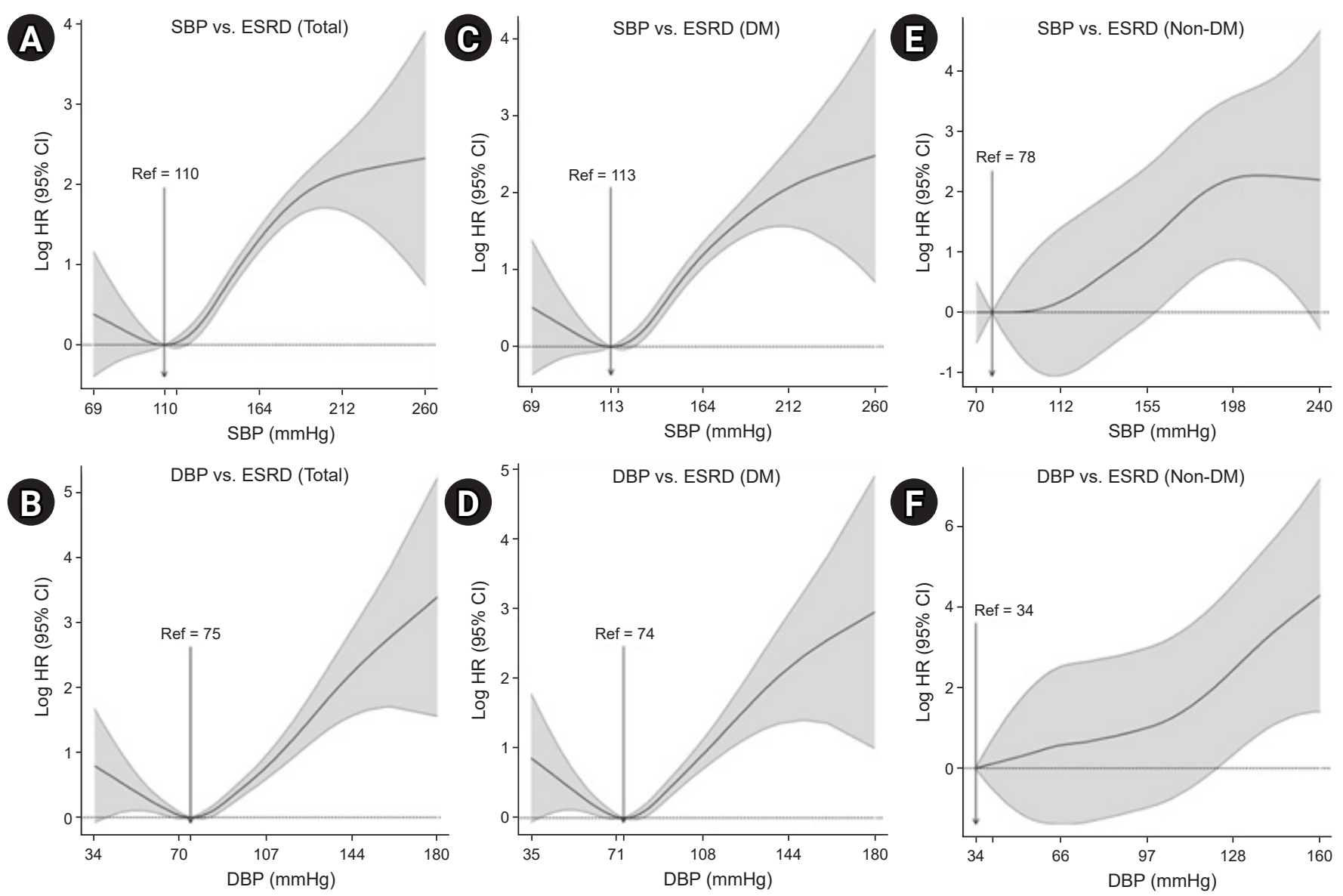

Figure 3. Cubic spline curves depicting the relationship between SBP or DBP and ESRD risk. (A, B) In total, (C, D) diabetes mellitus, and (D, F) non-DM subgroup. Adjusted for age, sex, income, DM, dyslipidemia, and hypertension, smoking, alcohol drinking, physical activity, and estimated glomerular filtration rate.

$\mathrm{Cl}$, confidence interval; DBP, diastolic blood pressure; DM, diabetes mellitus; ESRD, end-stage renal disease; HR, hazard ratio; SBP, systolic blood pressure; Ref, reference. 


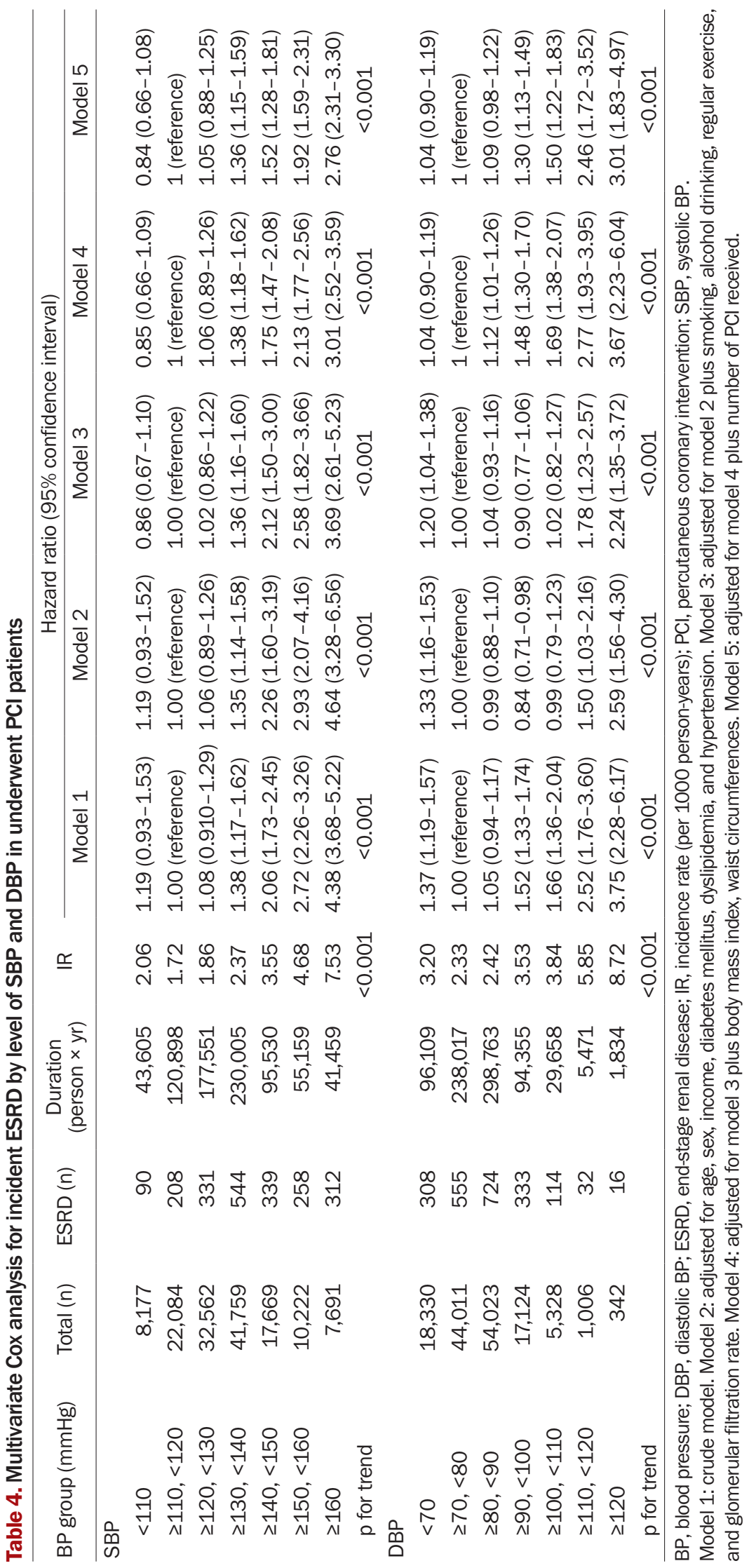


Table 5. Multivariate Cox analysis for incident ESRD by level of SBP and DBP in underwent PCI patients (subgroup analysis for DM)

\begin{tabular}{|c|c|c|c|c|c|c|c|}
\hline \multirow{2}{*}{ BP group (mmHg) } & \multirow{2}{*}{ Total $(n)$} & \multirow{2}{*}{$\operatorname{ESRD}(n)$} & \multirow{2}{*}{$\begin{array}{c}\text { Duration } \\
(\text { person } \times \text { yr) }\end{array}$} & \multirow{2}{*}{$\mathrm{IR}$} & \multicolumn{3}{|c|}{ Hazard ratio (95\% confidence interval) } \\
\hline & & & & & Model 1 & Model 2 & Model 3 \\
\hline \multicolumn{8}{|l|}{ SBP } \\
\hline \multicolumn{8}{|l|}{ Non-DM } \\
\hline$<110$ & 5,644 & 15 & 30,642 & 0.49 & $0.79(0.44-1.39)$ & $0.79(0.44-1.39)$ & $0.77(0.43-1.36)$ \\
\hline$\geq 110,<120$ & 15,370 & 53 & 85,321 & 0.62 & 1.00 (reference) & 1.00 (reference) & 1.00 (reference) \\
\hline$\geq 120,<130$ & 22,370 & 79 & 123,443 & 0.64 & $1.03(0.73-1.46)$ & $1.02(0.72-1.45)$ & $1.04(0.73-1.47)$ \\
\hline$\geq 130,<140$ & 28,401 & 162 & 158,921 & 1.02 & $1.64(1.21-2.24)$ & $1.60(1.17-2.19)$ & $1.64(1.20-2.24)$ \\
\hline$\geq 140,<150$ & 11,411 & 76 & 62,928 & 1.20 & $1.94(1.37-2.76)$ & $2.32(1.18-4.59)$ & $2.37(1.20-4.68)$ \\
\hline$\geq 150,<160$ & 6,532 & 60 & 36,089 & 1.66 & $2.68(1.85-3.87)$ & $3.12(1.57-6.23)$ & $3.20(1.60-6.38)$ \\
\hline$\geq 160$ & 4,796 & 75 & 26,808 & 2.80 & $4.51(3.18-6.42)$ & $5.01(2.53-9.91)$ & $5.14(2.60-10.18)$ \\
\hline \multicolumn{8}{|l|}{$\mathrm{DM}$} \\
\hline$<110$ & 2,533 & 75 & 12,963 & 5.79 & $1.32(1.00-1.74)$ & $1.32(1.00-1.74)$ & $1.31(0.99-1.73)$ \\
\hline$\geq 110,<120$ & 6,714 & 155 & 35,577 & 4.36 & 1 (reference) & 1 (reference) & 1 (reference) \\
\hline$\geq 120,<130$ & 10,192 & 252 & 54,108 & 4.66 & $1.07(0.88-1.31)$ & $1.07(0.88-1.31)$ & $1.09(0.89-1.33)$ \\
\hline$\geq 130,<140$ & 13,358 & 382 & 71,084 & 5.37 & $1.24(1.03-1.49)$ & $1.26(1.05-1.52)$ & $1.30(1.08-1.58)$ \\
\hline$\geq 140,<150$ & 6,258 & 263 & 32,602 & 8.07 & $1.85(1.52-2.25)$ & $2.23(1.50-3.33)$ & $2.28(1.53-3.41)$ \\
\hline$\geq 150,<160$ & 3,690 & 198 & 19,070 & 10.38 & $2.38(1.93-2.93)$ & $2.86(1.91-4.30)$ & $2.94(1.96-4.42)$ \\
\hline$\geq 160$ & 2,895 & 237 & 14,651 & 16.18 & $3.70(3.02-4.53)$ & $4.47(2.99-6.69)$ & $4.66(3.11-6.97)$ \\
\hline$p$ for interaction & & & & & 0.09 & 0.10 & 0.11 \\
\hline \multicolumn{8}{|l|}{ DBP } \\
\hline \multicolumn{8}{|l|}{ Non-DM } \\
\hline$<70$ & 11,515 & 60 & 61,701 & 0.97 & $1.10(0.82-1.49)$ & $1.04(0.77-1.41)$ & $1.03(0.76-1.40)$ \\
\hline$\geq 70,<80$ & 29,440 & 142 & 161,769 & 0.88 & 1.00 (reference) & 1.00 (reference) & 1.00 (reference) \\
\hline$\geq 80,<90$ & 37,331 & 200 & 209,254 & 0.96 & $1.09(0.88-1.35)$ & $1.06(0.85-1.31)$ & $1.06(0.85-1.32)$ \\
\hline$\geq 90,<100$ & 11,559 & 82 & 64,918 & 1.26 & $1.44(1.10-1.90)$ & $0.88(0.63-1.22)$ & $0.88(0.63-1.23)$ \\
\hline$\geq 100,<110$ & 3,727 & 23 & 21,201 & 1.09 & $1.24(0.80-1.93)$ & $0.83(0.51-1.34)$ & $0.83(0.51-1.34)$ \\
\hline$\geq 110,<120$ & 701 & 8 & 3,912 & 2.05 & $2.34(1.15-4.76)$ & $1.67(0.80-3.50)$ & $1.70(0.81-3.56)$ \\
\hline$\geq 120$ & 251 & 5 & 1,397 & 3.58 & $4.08(1.67-9.94)$ & $2.59(1.56-4.30)$ & $2.24(1.35-3.72)$ \\
\hline \multicolumn{8}{|l|}{$\mathrm{DM}$} \\
\hline$<70$ & 6,815 & 248 & 34,408 & 7.21 & $1.32(1.13-1.54)$ & $1.45(1.23-1.70)$ & $1.43(1.22-1.68)$ \\
\hline$\geq 70,<80$ & 14,571 & 413 & 76,248 & 5.42 & 1.00 (reference) & 1.00 (reference) & 1.00 (reference) \\
\hline$\geq 80,<90$ & 16,692 & 524 & 89,509 & 5.85 & $1.09(0.96-1.24)$ & $0.96(0.84-1.09)$ & $0.972(0.85-1.11)$ \\
\hline$\geq 90,<100$ & 5,565 & 251 & 29,437 & 8.53 & $1.58(1.35-1.85)$ & $0.81(0.68-0.98)$ & $0.83(0.69-1.00)$ \\
\hline$\geq 100,<110$ & 1,601 & 91 & 8,457 & 10.76 & $2.00(1.59-2.51)$ & $1.02(0.80-1.31)$ & $1.06(0.82-1.36)$ \\
\hline$\geq 110,<120$ & 305 & 24 & 1,559 & 15.39 & $2.84(1.88-4.29)$ & $1.40(0.91-2.14)$ & $1.51(0.98-2.30)$ \\
\hline$\geq 120$ & 91 & 11 & 437 & 25.20 & $4.60(2.53-8.37)$ & $2.33(1.27-4.29)$ & $2.65(1.44-4.88)$ \\
\hline $\mathrm{p}$ for trend & & & & & 0.55 & 0.34 & 0.32 \\
\hline
\end{tabular}

$\mathrm{BP}$, blood pressure; DBP, diastolic BP; DM, diabetes mellitus; ESRD, end-stage renal disease; IR, incidence rate (per 1000 person-years); $\mathrm{PCl}$, percutaneous coronary intervention; SBP, systolic BP.

Model 1: crude model. Model 2: adjusted for age, sex, income, DM, dyslipidemia, and hypertension. Model 3: adjusted for model 2 plus smoking, alcohol drinking, regular exercise, and glomerular filtration rate.

$\geq 140 \mathrm{mmHg}$, achieving < $140 \mathrm{mmHg}$ produced significant benefits in the $\mathrm{CV}$ outcome, but there was no further benefit at lower SBP levels [18].

Recent post hoc and secondary analyses of SPRINT have shown that patients with low baseline CV risk had less ben- efit and more adverse renal events in the intensively treated group than in the standard group [19]. Furthermore, those patients in the lowest quintile of DBP at baseline $(61 \mathrm{mmHg})$ had higher rates of CV events, but intensive lowering of SBP in this group was still beneficial relative to the low DBP 
Table 6. Multivariate Cox analysis for incident ESRD by level of SBP and DBP in underwent PCI patients (subgroup analysis for CKD)

\begin{tabular}{|c|c|c|c|c|c|c|c|}
\hline \multirow{2}{*}{ BP group (mmHg) } & \multirow{2}{*}{ Total $(n)$} & \multirow{2}{*}{$\operatorname{ESRD}(n)$} & \multirow{2}{*}{$\begin{array}{c}\text { Duration } \\
\text { (person } \times \text { yr) }\end{array}$} & \multirow{2}{*}{ IR } & \multicolumn{3}{|c|}{ Hazard ratio (95\% confidence interval) } \\
\hline & & & & & Model 1 & Model 2 & Model 3 \\
\hline \multicolumn{8}{|l|}{ SBP } \\
\hline \multicolumn{8}{|l|}{ Non-CKD } \\
\hline$<110$ & 6,907 & 20 & 37,378 & 0.54 & $1.16(0.69-1.95)$ & $1.18(0.70-1.98)$ & $1.16(0.69-1.96)$ \\
\hline$\geq 110,<120$ & 19,127 & 49 & 105,460 & 0.46 & 1.00 (reference) & 1.00 (reference) & 1.00 (reference) \\
\hline$\geq 120,<130$ & 28,120 & 77 & 154,889 & 0.50 & $1.07(0.75-1.53)$ & $1.05(0.73-1.50)$ & $1.07(0.74-1.53)$ \\
\hline$\geq 130,<140$ & 35,854 & 126 & 199,710 & 0.63 & $1.35(0.97-1.88)$ & $1.32(0.94-1.84)$ & $1.36(0.97-1.90)$ \\
\hline$\geq 140,<150$ & 14,561 & 62 & 80,119 & 0.77 & $1.67(1.15-2.42)$ & $1.81(0.88-3.71)$ & $1.87(0.91-3.82)$ \\
\hline$\geq 150,<160$ & 8,350 & 45 & 46,148 & 0.98 & $2.09(1.40-3.14)$ & $2.26(1.09-4.72)$ & 2.35 (1.13-4.89) \\
\hline$\geq 160$ & 6,024 & 44 & 33,670 & 1.31 & $2.79(1.86-4.20)$ & $2.95(1.41-6.15)$ & 3.07 (1.47-6.41) \\
\hline \multicolumn{8}{|l|}{ CKD } \\
\hline$<110$ & 1,270 & 70 & 6,227 & 11.24 & $1.07(0.81-1.42)$ & $1.02(0.77-1.35)$ & $1.00(0.76-1.33)$ \\
\hline$\geq 110,<120$ & 2,957 & 159 & 15,437 & 10.30 & 1.00 (reference) & 1.00 (reference) & 1.00 (reference) \\
\hline$\geq 120,<130$ & 4,442 & 254 & 22,662 & 11.21 & $1.08(0.89-1.32)$ & $1.11(0.91-1.36)$ & $1.12(0.92-1.36)$ \\
\hline$\geq 130,<140$ & 5,905 & 418 & 30,294 & 13.80 & 1.33 (1.11-1.60) & 1.41 (1.17-1.69) & $1.42(1.18-1.70)$ \\
\hline$\geq 140,<150$ & 3,108 & 277 & 15,411 & 18.00 & $1.73(1.42-2.10)$ & 2.39 (1.61-3.54) & $2.36(1.59-3.50)$ \\
\hline$\geq 150,<160$ & 1,872 & 213 & 9,012 & 23.64 & $2.25(1.83-2.77)$ & $2.93(1.97-4.38)$ & $2.91(1.95-4.33)$ \\
\hline$\geq 160$ & 1,667 & 268 & 7,789 & 34.41 & 3.28 (2.69-3.99) & $4.71(3.17-6.99)$ & $4.65(3.13-6.90)$ \\
\hline$p$ for interaction & & & & & 0.98 & 0.94 & 0.97 \\
\hline \multicolumn{8}{|l|}{ DBP } \\
\hline \multicolumn{8}{|l|}{ Non-CKD } \\
\hline$<70$ & 14,929 & 50 & 79,703 & 0.63 & $1.03(0.74-1.44)$ & $1.03(0.74-1.44)$ & $1.025(0.734-1.430)$ \\
\hline$\geq 70,<80$ & 37,519 & 117 & 205,455 & 0.57 & 1.00 (reference) & 1.00 (reference) & 1.00 (reference) \\
\hline$\geq 80,<90$ & 46,407 & 156 & 259,319 & 0.60 & $1.08(0.85-1.37)$ & $1.08(0.85-1.37)$ & 1.09 (0.85-1.39) \\
\hline$\geq 90,<100$ & 14,433 & 72 & 80,966 & 0.89 & 1.30 (0.88-1.91) & $1.30(0.88-1.91)$ & $1.30(0.89-1.92)$ \\
\hline$\geq 100,<110$ & 4,528 & 19 & 25,652 & 0.74 & 1.18 (0.68-2.03) & $1.18(0.68-2.03)$ & 1.19 (0.69-2.05) \\
\hline$\geq 110,<120$ & 857 & 5 & 4,788 & 1.04 & 1.65 (0.65-4.19) & 1.65 (0.65-4.19) & $1.69(0.67-4.30)$ \\
\hline$\geq 120$ & 270 & 4 & 1,490 & 2.68 & $4.91(1.75-13.75)$ & $4.91(1.75-13.75)$ & $5.02(1.79-14.09)$ \\
\hline \multicolumn{8}{|l|}{ CKD } \\
\hline$<70$ & 3,401 & 258 & 16,407 & 15.73 & $1.25(1.07-1.46)$ & $1.25(1.07-1.46)$ & 1.25 (1.07-1.46) \\
\hline$\geq 70,<80$ & 6,492 & 438 & 32,561 & 13.45 & 1.00 (reference) & 1.00 (reference) & 1.00 (reference) \\
\hline$\geq 80,<90$ & 7,616 & 568 & 39,444 & 14.40 & $0.98(0.86-1.11)$ & $0.98(0.86-1.11)$ & $0.98(0.87-1.11)$ \\
\hline$\geq 90,<100$ & 2,691 & 261 & 13,389 & 19.49 & $0.83(0.70-1.00)$ & $0.83(0.70-1.00)$ & $0.84(0.70-1.00)$ \\
\hline$\geq 100,<110$ & 800 & 95 & 4,005 & 23.72 & $0.96(0.75-1.22)$ & $0.96(0.75-1.22)$ & $0.98(0.77-1.24)$ \\
\hline$\geq 110,<120$ & 149 & 27 & 683 & 39.52 & $1.63(1.09-2.43)$ & $1.63(1.09-2.43)$ & 1.69 (1.13-2.53) \\
\hline$\geq 120$ & 72 & 12 & 344 & 34.89 & $1.32(0.74-2.37)$ & $1.32(0.74-2.374)$ & $1.43(0.80-2.57)$ \\
\hline $\mathrm{p}$ for trend & & & & & 0.70 & 0.64 & 0.65 \\
\hline
\end{tabular}

BP, blood pressure; CKD, chronic kidney disease; DBP, diastolic BP; ESRD, end-stage renal disease; IR, incidence rate (per 1000 person-years); PCI, percutaneous coronary intervention; SBP, systolic BP.

Model 1: crude model. Model 2: adjusted for age, sex, income, diabetes mellitus, dyslipidemia, and hypertension. Model 3: adjusted for model 2 plus smoking, alcohol drinking, regular exercise, and glomerular filtration rate.

group [20]. A linear relationship between levels of BP and $\mathrm{CV}$ outcomes has been observed in the general hypertensive population, particularly for stroke; however, in patients with coronary artery disease, the relationship of BP and CV outcomes often shows a J-shaped curve with higher CV event rates at lower levels of $\mathrm{BP}[21]$.

Several pathophysiological mechanisms have been proposed to explain the existence of a J-shaped curve. The J-shaped curve may represent an epiphenomenon of increased arterial stiffness; thus, a low DBP level might be 
a marker of high pulse pressure and increased mortality because coronary perfusion occurs during diastole [22]. In our analyses, we noticed a J-shaped curve phenomenon just for DBP but not for SBP in patients with DM, for which the pulse pressure theory would be applicable. However, the hypothesis that a J-shaped curve might be an epiphenomenon of severe underlying chronic illness or inflammation seems more convincing.

Unfortunately, there is no published research on the measurement of BP during health check-ups prior to PCI and ESRD prognosis after PCI. High SBP increases cardiac afterload, whereas low DBP may lead to impaired coronary perfusion. Therefore, the higher the pulse pressure immediately before PCI, the worse the prognosis after PCI [23]. However, it is difficult to determine whether the previous study's mechanism is the same as that of the current study due to different participant characteristics and methodology. Further research on the mechanism of why BP measured within two years prior to PCI affects the progression to ESRD after PCI is warranted. However, high BP progressively leads to the development of acute coronary syndrome; thus, it can be considered an ESRD progression factor.

This study has several limitations. First, the study population comprised Korean men and women; hence, it is uncertain whether these findings can be generalized to other ethnic groups. Second, different BP devices were used to measure BP, and no standardized protocols were used in each center. Third, antihypertensive drugs, such as renin-angiotensin-aldosterone system blockers, are known to delay the progression of CKD, but these antihypertensive medication effects were not considered in this study. Fourth, there are various time differences between BP measurement and PCI. The maximal time difference was two years, and the average time difference was $1.04 \pm 0.57$ years. However, the time difference effects were not considered in this study.

In conclusion, our study showed that a high SBP or DBP prior to PCI was independently associated with an increased incidence of ESRD and low DBP also risk for ESRD in PCI patients with DM.

\section{Conflicts of interest}

All authors have no conflicts of interest to declare.

\section{Funding}

This research was supported by grant of the Korea Health Technology R\&D Project through the Korea Health Industry Development Institute (KHIDI), funded by the Ministry of Health \& Welfare, Republic of Korea (grant number: HI18C0331, HR20C0021), and by a grant (BCRI20025\&20076) of Chonnam National University Hospital Biomedical Research Institute.

\section{Authors' contributions}

Conceptualization: EHB, SYL

Data curation: BK, KDH

Formal analysis: TRO, HSC

Funding acquisition: SWK

Investigation: $\mathrm{BK}, \mathrm{KDH}$

Project administration: EHB, SYL

Visualization: CSK, SKM

Writing-original draft: EHB, SYL

Writing-review \& editing: CSK, SKM

All authors read and approved the final manuscript.

\section{ORCID}

Eun Hui Bae, https://orcid.org/0000-0003-1727-2822

Sang Yup Lim, https://orcid.org/0000-0002-3042-6702

Bongseong Kim, https://orcid.org/0000-0002-1022-3553

Kyung-Do Han, https://orcid.org/0000-0003-2718-4308

Tae Ryom Oh, https://orcid.org/0000-0002-3713-0939

Hong Sang Choi, https://orcid.org/0000-0001-8191-4071

Chang Seong Kim, https://orcid.org/0000-0001-8753-7641

Seong Kwon Ma, https://orcid.org/0000-0002-5758-8189

Soo Wan Kim, https://orcid.org/0000-0002-3540-9004

\section{References}

1. GBD 2015 Risk Factors Collaborators. Global, regional, and national comparative risk assessment of 79 behavioural, environmental and occupational, and metabolic risks or clusters of risks, 1990-2015: a systematic analysis for the Global Burden of Disease Study 2015. Lancet 2016;388:1659-1724.

2. Mancia G, Fagard R, Narkiewicz K, et al. 2013 ESH/ESC guidelines for the management of arterial hypertension: the Task Force for the Management of Arterial Hypertension of the Euro- 
pean Society of Hypertension (ESH) and of the European Society of Cardiology (ESC). Eur Heart J 2013;34:2159-2219.

3. Rosendorff C, Lackland DT, Allison M, et al. Treatment of hypertension in patients with coronary artery disease: a scientific statement from the American Heart Association, American College of Cardiology, and American Society of Hypertension. Circulation 2015;131:e435-e470.

4. Chobanian AV, Bakris GL, Black HR, et al. The seventh report of the Joint National Committee on Prevention, Detection, Evaluation, and Treatment of High Blood Pressure: the JNC 7 report. JAMA 2003;289:2560-2572.

5. Lewington S, Clarke R, Qizilbash N, Peto R, Collins R; Prospective Studies Collaboration. Age-specific relevance of usual blood pressure to vascular mortality: a meta-analysis of individual data for one million adults in 61 prospective studies. Lancet 2002;360:1903-1913.

6. Cruickshank JM, Thorp JM, Zacharias FJ. Benefits and potential harm of lowering high blood pressure. Lancet 1987;1:581-584.

7. Lindholm L, Lanke J, Bengtsson B, Ejlertsson G, Thulin T, Scherstén B. Both high and low blood pressures risk indicators of death in middle-aged males. Isotonic regression of blood pressure on age applied to data from a 13-year prospective study. Acta Med Scand 1985;218:473-480.

8. Messerli FH, Mancia G, Conti CR, et al. Dogma disputed: can aggressively lowering blood pressure in hypertensive patients with coronary artery disease be dangerous? Ann Intern Med 2006;144:884-893.

9. Stewart IM. Relation of reduction in pressure to first myocardial infarction in patients receiving treatment for severe hypertension. Lancet 1979;1:861-865.

10. Bangalore S, Qin J, Sloan S, Murphy SA, Cannon CP; PROVE ITTIMI 22 Trial Investigators. What is the optimal blood pressure in patients after acute coronary syndromes?: relationship of blood pressure and cardiovascular events in the PRavastatin OR atorVastatin Evaluation and Infection Therapy-Thrombolysis In Myocardial Infarction (PROVE IT-TIMI) 22 trial. Circulation 2010;122:2142-2151.

11. White WB, Jalil F, Cushman WC, et al. Average clinician-measured blood pressures and cardiovascular outcomes in patients with type 2 diabetes mellitus and ischemic heart disease in the EXAMINE trial. J Am Heart Assoc 2018;7:e009114.

12. Lee J, Lee JS, Park SH, Shin SA, Kim K. Cohort profile: the National Health Insurance Service-National Sample Cohort (NHISNSC), South Korea. Int J Epidemiol 2017;46:e15.

13. Lee YH, Han K, Ko SH, Ko KS, Lee KU; Taskforce Team of Diabe- tes Fact Sheet of the Korean Diabetes Association. Data analytic process of a nationwide population-based study using national health information database established by national health insurance service. Diabetes Metab J 2016;40:79-82.

14. Yang HK, Han K, Kwon HS, et al. Obesity, metabolic health, and mortality in adults: a nationwide population-based study in Korea. Sci Rep 2016;6:30329.

15. Whelton PK, Carey RM, Aronow WS, et al. 2017 ACC/AHA/ AAPA/ABC/ACPM/AGS/APhA/ASH/ASPC/NMA/PCNA guideline for the prevention, detection, evaluation, and management of high blood pressure in adults: executive summary. A report of the American College of Cardiology/American Heart Association Task Force on Clinical Practice Guidelines. Hypertension 2018;71:1269-1324.

16. SPRINT Research Group; Wright JT Jr, Williamson JD, et al. A randomized trial of intensive versus standard blood-pressure control. N Engl J Med 2015;373:2103-2116.

17. Böhm M, Schumacher H, Teo KK, et al. Achieved blood pressure and cardiovascular outcomes in high-risk patients: results from ONTARGET and TRANSCEND trials. Lancet 2017;389:22262237.

18. Weber MA, Bloch M, Bakris GL, et al. Cardiovascular outcomes according to systolic blood pressure in patients with and without diabetes: an ACCOMPLISH substudy. J Clin Hypertens (Greenwich) 2016;18:299-307.

19. Phillips RA, Xu J, Peterson LE, Arnold RM, Diamond JA, Schussheim AE. Impact of cardiovascular risk on the relative benefit and harm of intensive treatment of hypertension. J Am Coll Cardiol 2018;71:1601-1610.

20. Beddhu S, Chertow GM, Cheung AK, et al. Influence of baseline diastolic blood pressure on effects of intensive compared with standard blood pressure control. Circulation 2018;137:134-143.

21. Samuelsson OG, Wilhelmsen LW, Pennert KM, Wedel H, Berglund GL. The J-shaped relationship between coronary heart disease and achieved blood pressure level in treated hypertension: further analyses of 12 years of follow-up of treated hypertensives in the Primary Prevention Trial in Gothenburg, Sweden. J Hypertens 1990;8:547-555.

22. Kannel WB, Wilson PW, Nam BH, D'Agostino RB, Li J. A likely explanation for the J-curve of blood pressure cardiovascular risk. Am J Cardiol 2004;94:380-384.

23. Warren J, Nanayakkara S, Andrianopoulos N, et al. Impact of pre-procedural blood pressure on long-term outcomes following percutaneous coronary intervention. J Am Coll Cardiol 2019;73:2846-2855. 